\title{
A novel technique for light delivery through branched or bent anatomic structures
}

\author{
Joseph S. Friedberg, MD \\ Cynthia Skema, BS ${ }^{b}$ \\ Jeffrey Burdick, BS \\ Arjun G. Yodh, PhD' \\ Shamus R. Carr, MD \\ Joseph P. Culver, $\mathrm{PhD}^{\mathrm{d}}$
}

From Thomas Jefferson University Hospital, ${ }^{\mathrm{a}}$ Philadelphia, Pa; Duke University, ${ }^{\mathrm{b}}$ Durham, NC; University of Pennsylvania, ${ }^{c}$ Philadelphia, Pa; and Harvard Medical School, ${ }^{\mathrm{d}}$ Boston, Mass.

Received for publication May 1, 2003; revisions requested June 2, 2003; revisions received June 23, 2003; accepted for publication July 7, 2003.

Address for reprints: Joseph S. Friedberg, MD, 1025 Walnut St, Suite 605, Philadelphia, PA 19107 (E-mail: Joseph.Friedberg @jefferson.edu).

J Thorac Cardiovasc Surg 2003;126:1963-7

Copyright $\odot 2003$ by The American Association for Thoracic Surgery

$0022-5223 / 2003 \$ 30.00+0$

doi: $10.1016 / \mathrm{S} 0022-5223(03) 01320-5$
Objective: Photodynamic therapy is an effective cancer treatment, but light delivery constraints currently limit its application to superficial, easily visualized tumors. The goal of this study was to determine whether it would be possible to manipulate the optical properties of irregularly shaped anatomic structures for the purpose of light delivery. Such a technique could potentially expand the role of photodynamic therapy to treat tumors currently viewed as inaccessible to visible light.

Methods: Ex vivo sheep tracheas and lungs were filled with substances of varying refractive indices. The effects on transmission of visible light of a known wavelength introduced into the proximal lumen of the organs were studied. Data were collected with naked-eye observation, standard photography, charge-coupled device imaging, and direct light measurement.

Results: Filling a lung or trachea with a liquid possessing a refractive index higher than that of tissue dramatically increases the ability to deliver light around bends and through a branched network.

Conclusion: It is possible to manipulate the optical properties of an ex vivo organ for the purpose of enhanced light delivery.

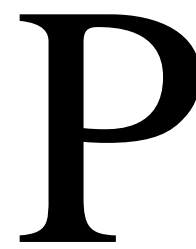
hotodynamic therapy (PDT) is a technique for killing cancer cells that involves activating a photosensitizer with visible light in the presence of oxygen. PDT is very effective but is currently limited to the treatment of thin, easily visualized tumors because of the limited penetration of visible light into tissue and the necessity for direct application of the light. ${ }^{1-10}$ Treatment of lung cancer, for instance, is essentially limited to treating endobronchial tumors that can be visualized through a bronchoscope. ${ }^{11,12}$

There are cancers of the lung, such as diffuse bronchoalveolar carcinoma, for which no effective treatment currently exists. Bronchoalveolar carcinoma lines the alveoli and bronchioles, forming a thin layer of cancer cells in the terminal portion of the bronchial tree. ${ }^{13-16}$ Similarly, innumerable small pulmonary metastases, depending on the identity of the primary tumor, might also represent an untreatable disease. ${ }^{17-21}$ If it was possible to deliver light throughout an entire lung, PDT could potentially be used to treat these diseases.

Total internal reflection is the principle that is capitalized on to allow fiberoptic cables to be used as "light pipes" to deliver light over many miles. Internal reflection is favored when the refractive index (RI) of the lumen of a tube is greater than the RI of the wall of the tube. Our hypothesis is that if it was possible to use the luminal network of an organ for light piping, then it might be possible to deliver light throughout an entire organ, perhaps making PDT a treatment option for diffuse 

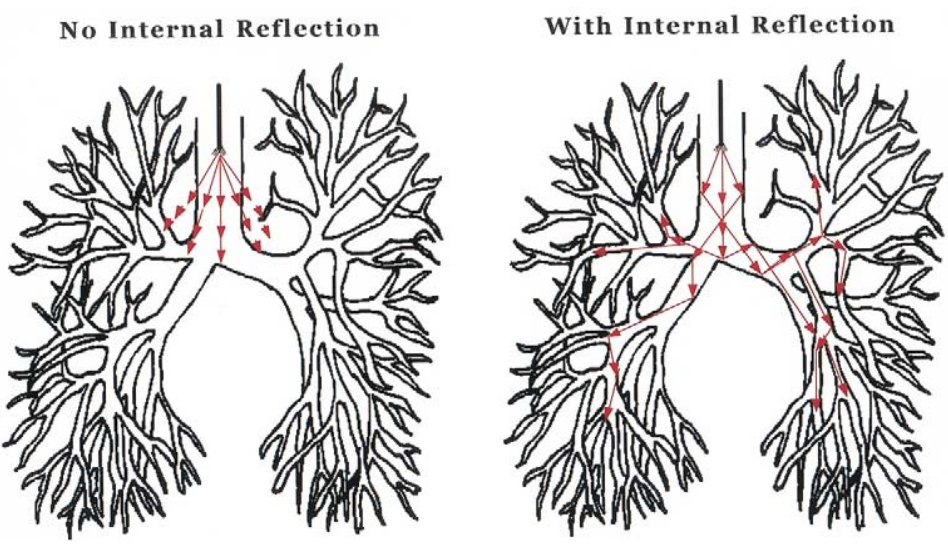

Figure 1. Schematic diagram of light-piping hypothesis. The left panel depicts light introduced into an unaltered proximal airway where the lumen is of an RI lower than that of tissue, demonstrating limited penetration and no propagation. The right pane/ depicts light entering an airway where the lumen has an RI higher than that of tissue, favoring internal reflection and distal light piping.

cancers in that organ (Figure 1). Some organs, such as the intestines, might have bends and folds, but the lumen is a simple tube. The lumen of other organs, such as the lung, is comprised of a complex branched network. What follows is the first description of the light-piping phenomenon as applied to anatomic structures, both simple and branched.

\section{Materials and Methods}

\section{Organ Harvesting and Preparation}

Lung blocks, including the full length of the trachea, were harvested from sheep (approximately 50-100 kg) in a US Department of Agriculture-approved Halal slaughterhouse (Trenton, NJ). Sheep lungs were used because they resemble human lungs both macroscopically and microscopically. ${ }^{22}$ For tracheal experiments, the trachea was divided distally at the takeoff of the right upper lobe bronchus. For lung experiments, the right upper lobe and right mainstem bronchi were divided with a GIA stapling device (US Surgical Corp, Norwalk, Conn), leaving the left lung and trachea in continuity.

The lungs were kept at $4^{\circ} \mathrm{C}$ in saline solution that was changed daily. They were observed until the entire lung was atelectatic, a process requiring 1 to 5 days. Once this was achieved, the proximal trachea was tied with a heavy string in an airtight manner around a bronchoscopy adapter (DHD Healthcare, Canastota, NY). The portion of the adapter that connects to airway tubing was sealed in an airtight manner, leaving only the bronchoscopy valve accessible.

\section{Light Source and Delivery}

Red light, $630 \mathrm{~nm}$ at a power of $0.1 \mathrm{~W}$, was supplied from a tunable dye laser (Laserscope, San Jose, Calif) and delivered through a 1-mm-flat cut fiber (Rare Earth Medical Inc, West Yarmouth, Mass).

\section{Inflational Media}

By using an RI of 1.37 for tissue, air (RI, 1.00), saline solution (RI, 1.33), and mineral oil (RI, 1.46) were selected for luminal filling to study the effect of lower, matched, and higher luminal RIs. ${ }^{23}$

\section{Photographs}

Photodocumentation of lung and trachea experiments was obtained with both a 35-mm camera using 400-speed color film and a CCD camera (Model NTE/CCD-1340/1300; Roper Scientific, Vianen, The Netherlands) at an f-stop of 8 and 3 seconds' exposure time.

\section{Trachea Experiments}

Tracheas (10-13 cm in length) were bent into a $U$ shape, immersed in $10 \%$ intralipid to prevent any errant light guiding that might result from an external trachea-air interface, and supported on a ring stand. The 2 limbs were separated with a piece of opaque foam. The bent tracheas were filled with air, saline solution, or mineral oil. Then a laser light was shined into the opening at one end, and photo documentation was obtained. Quantitative measurements were made by pulling an isotropic light detector (University of Pennsylvania, Department of Radiation Oncology) through the entire length of the trachea and making serial measurements of the measured fluence at each location.

\section{Lung Experiments}

The lung was suspended from a ring stand and draped in opaque cloth, such that only the lung, but not the trachea, was visible. By using the bronchoscopy adaptor for access, lungs were then filled with air, saline solution, or mineral oil. A bronchoscope (Olympus America Inc, Melville, NY) was used to position the light fiber in the distal trachea $2 \mathrm{~cm}$ above the carina. Laser light was then shined into the lung, and photo documentation was obtained. Images were also captured with the CCD camera for quantitative estimations. 

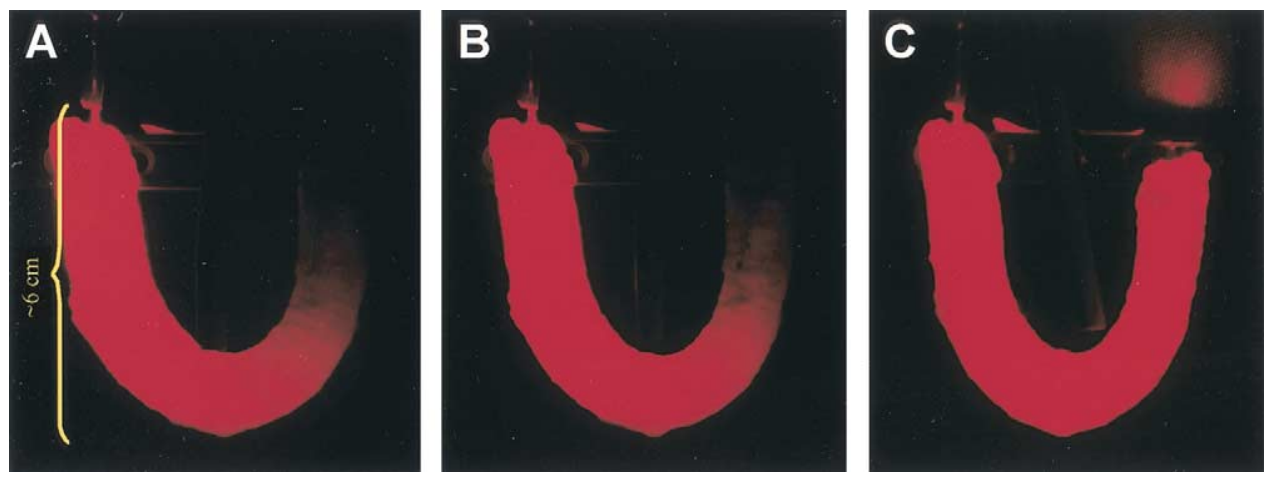

Figure 2. Bent tracheal experiment: results of $630 \mathrm{~nm}$ of light delivered through tracheas bent $180^{\circ}$. $A$ is the trachea filled with air. $B$ is the trachea filled with saline solution. $C$ is the trachea filled with mineral oil. Notice the dramatic enhancement of light delivery with mineral oil when the lumen possesses an RI higher than that of the tracheal wall.

\section{Results}

\section{Bent Tracheal Experiments}

Representative photographs of $630 \mathrm{~nm}$ of light delivered through the tracheas bent $180^{\circ}$ into a $\mathrm{U}$ shape are shown in Figure 2. As viewed with the naked eye, the proximal limb of the U glowed bright red, regardless of what substance was in the lumen. For both air and saline solution, the distal limb of the $\mathrm{U}$ appeared illuminated only to the bend at the bottom of the $\mathrm{U}$ (Figure 2, $A$ and $B$ ). Filling the trachea with mineral oil, however, resulted in both limbs of the U appearing equally bright and with light shining out from the distal opening (Figure 2, C).

Quantitative evaluation of light propagation through the bent tracheas revealed similar light intensities in the proximal half of all the tracheas before the $180^{\circ}$ turn (Figure 3). After the midpoint of the tracheas filled with air and saline solution was reached, there was a rapid, 6-log intensity decrease. The trachea filled with mineral oil, however, demonstrated only a 2-log decrease after the bend in the trachea, followed by a plateau. Compared with air or saline solution, mineral oil resulted in a 4-log increase in distal light delivery.

\section{Lung Experiments}

Representative photographs of the results obtained when $630 \mathrm{~nm}$ of light was introduced into ex vivo sheep lungs with the light source positioned $2 \mathrm{~cm}$ above the trachea are shown in Figure 4. When the lung was inflated with air, it was barely possible to discern with the naked eye that light was being shined into the lung. There was only a suggestion of light transmission corresponding to the light fiber tip (Figure 4, A). The saline-filled lung demonstrated a small area of diffuse transillumination centered about the fiber tip (Figure 4, B). When the lung was filled with mineral oil, the entire lung revealed bright, dramatic, and near-uniform illumination (Figure 4,C).
CCD pictures allowed quantitation of the light intensity at similar areas of the lung surfaces. These areas were integrated to yield an average fluence for that portion of the lung. The average fluence densities for air, saline solution, and mineral oil were 55, 132, and $8861 \mathrm{nW} / \mathrm{cm}^{2}$, respectively.

\section{Discussion}

PDT is used for the treatment of many malignancies. The ability to treat these cancers is contingent on the lesion being thin enough to be penetrated with visible light and also accessible for direct illumination. There are many areas in the body at which there are cancers that could potentially be treated with PDT but are currently viewed as inaccessible for light delivery.

We hypothesized that introducing a liquid into an organ that increases the RI of the lumen of that organ beyond that of its walls would favor internal reflection and convert the lumen into light pipes similar to fiberoptic cables. Using an ex vivo trachea bent $180^{\circ}$, we demonstrated that light can be made to follow the lumen of a simple tube around a sharp bend. A much more complex model is that of an extensively branched network. Using an ex vivo lung inflated with mineral oil, we demonstrated that light can be introduced into the trachea, the most proximal trunk of the network, and that it follows the airways all the way to the surface of the lung, thereby illuminating the entire lung. This phenomenon only occurred when the lung was filled with mineral oil, a material with an RI higher than that of tissue, but not when the RI of the inflational medium was matched or lower than that of tissue.

Although the purpose of these experiments was primarily qualitative, an attempt was made to quantitate some aspects of the observed phenomenon. We measured a 4-log increase in the light reaching the distal end of the mineral oil-filled 


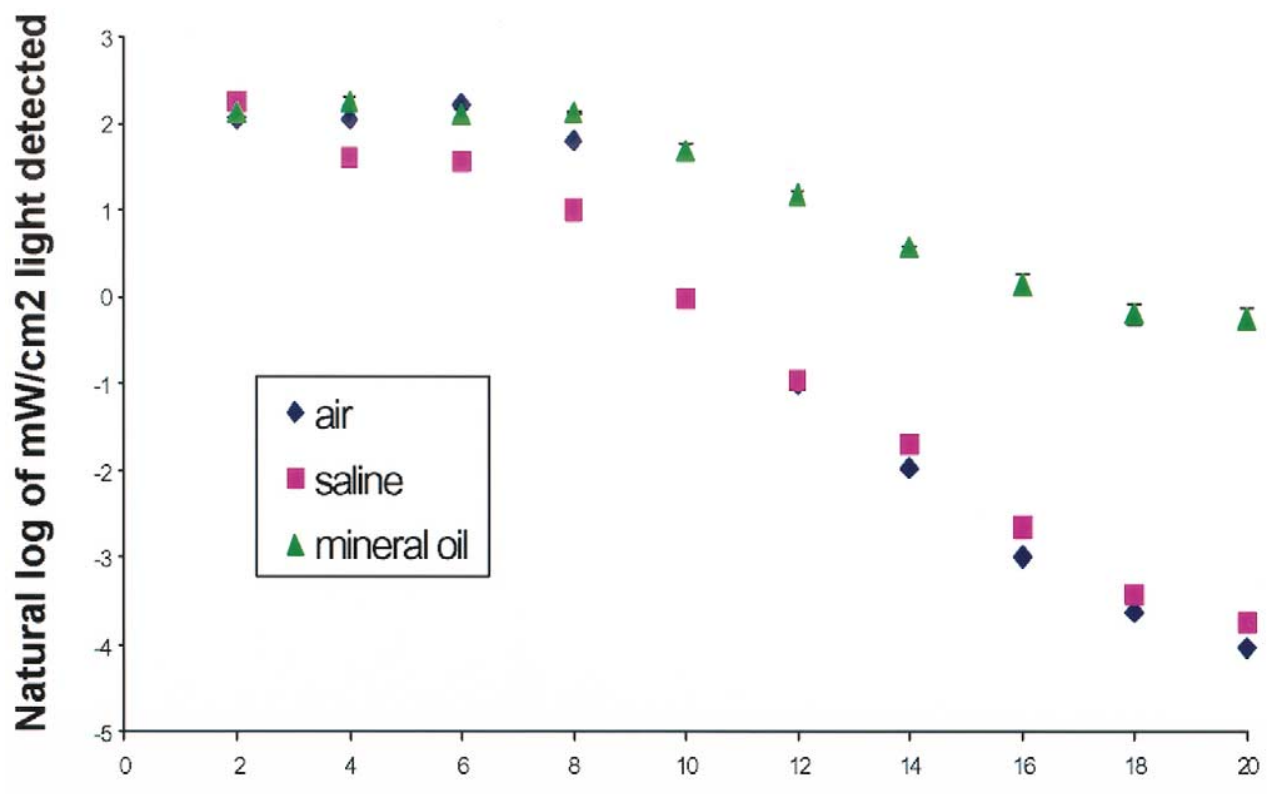

\section{Distance of isotropic detector from light source $(\mathrm{cm})$}

Figure 3. Light propagation at varying distances in a sheep trachea (bent $180^{\circ}$ ) filled with air, saline solution, or mineral oil. Error bars shown for mineral oil are $\pm 1 \mathrm{SE}$.
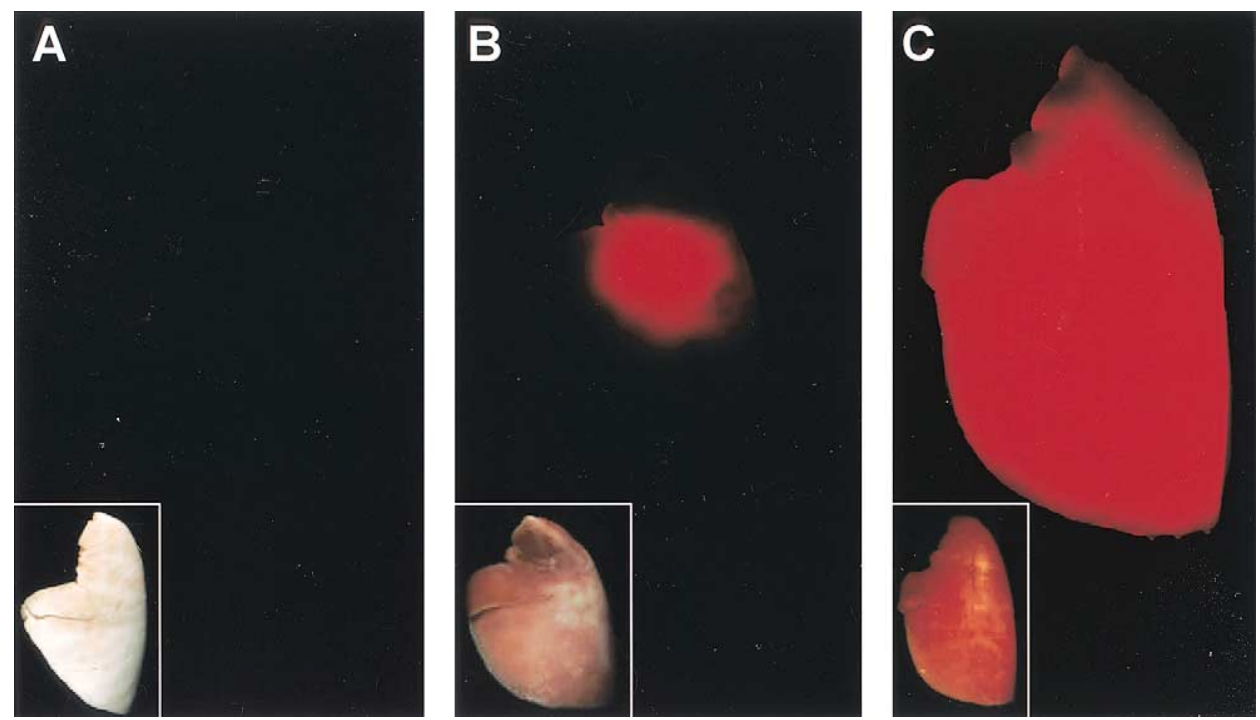

Figure 4. Ex vivo lungs: results of $630 \mathrm{~nm}$ of light delivered from a point $2 \mathrm{~cm}$ above the carina into lungs filled with media of different RIs. $A$ shows the results with air as the inflation media. $B$ shows the results with saline solution as the inflation media. $C$ shows the results of the lung filled with mineral oil. Small insets show the appearance of the lungs with the room lights on, and large panels show the appearance of the lungs in a dark room.

trachea when compared with the air- or saline-filled tracheas. The CCD estimations of light delivery to the surface of the lung with mineral oil for the measured area was more than 60 times that measured with saline and 160 times that for air. It should be noted, however, that this is a very crude estimation given that it is derived from a 2-dimensional image of one surface of a complexly shaped 3-dimensional object.

These proof-of-principle experiments demonstrate that it is possible to deliver light throughout an organ, either a 
simple tube bent on itself or a complex branched network. We speculate that if the proper substances were used to fill an organ, then visible light could be delivered, and cancers within that organ could potentially be treated with PDT. Furthermore, because light need only be introduced proximally, it is possible that any anatomic region that can be accessed percutaneously or endoscopically could be manipulated for light delivery. Thus in addition to the lungs, it is conceivable that the bowel lumen, biliary ducts, pancreatic ducts, urinary tract, sinuses, peritoneum, or even the cerebrospinal space could all be accessed with this technique for light delivery to their respective organs or spaces. Furthermore, use of the vascular system could potentially permit access to any location for the purpose of light delivery.

In summary, these experiments demonstrate that the optical properties of an ex vivo trachea and lung can be manipulated for the purpose of light delivery. Such manipulation could potentially allow PDT to emerge as a treatment for certain tumors currently viewed as inaccessible to light. Further studies are underway to better understand and characterize the observed light-piping phenomenon and to adapt and refine the materials and methods for in vivo experimentation.

We gratefully acknowledge the support of the University of Pennsylvania Department of Radiation Oncology, as well as the technical assistance of Theodor Vulcan, Timothy Zhu, and Tracey Sims.

\section{References}

1. Vrouenraets M, Visser G, Snow G, van Dongen G. Basic principles, applications in oncology and improved selectivity of photodynamic therapy. Anticancer Res. 2003;23:505-22.

2. Korbelik M, Sun J, Zeng H. Ischaemia-reperfusion injury in photodynamic therapy-treated mouse tumours. Br J Cancer. 2003;88(5):760-6.

3. Wilson BD, Mang T. Photodynamic therapy for cutaneous malignancies. Clin Dermatol. 1995;13:91-6.

4. Valduga G, Reddi E, Garbisa S, Jori G. Photosensitization of cells with different metastatic potentials by liposome-delivered $\mathrm{Zn}$ (II)-phthalocyanine. Int J Cancer. 1998;75:412-7.

5. Richter AM, Kelly B, Liu JCDJ, Towers GHN, Dolphin D, Levy JG.
Preliminary studies on a more effective phototoxic agent than hematoporphyrin. J Natl Cancer Inst. 1987;79(6):1327-31.

6. Okunaka T, Kato H, Konaka C, Furukawa K, Harada M, Yamamoto Y. Photodynamic therapy of lung cancer with bronchial artery infusion of photofrin. Diagn Ther Endosc. 1996;2:203-6.

7. Jori G. Tumour photosensitizers: approaches to enhance the selectivity and efficiency of photodynamic therapy. J Photochem Photobiol B. 1996;36:87-93.

8. Iinuma S, Farshi SS, Ortel B, Hasan T. A mechanistic study of cellular photodestruction with 5-aminolaevulinic acid-induced porphyrin. $\mathrm{BrJ}$ Cancer. 1994;70:21-8.

9. Kolarova H, Ditrichova D, Wagner J. Penetration of the laser light into the skin in vitro. Lasers Surg Med. 1999;24:231-5.

10. vanHillegersberg R, vandenBerg JWO, Kort WJ, Terpstra OT, Wilson JHP. Selective accumulation of endogenously produced porphyrins in a liver metastasis model in rats. Gastroenterology. 1992;103:647-51.

11. Kato H. Photodynamic therapy for lung cancer-a review of 19 years experience. J Photochem. 1998;42:96-9.

12. Kato H. Photodynamic therapy for early stage central type of lung cancer. Mayo Found Med Educ Res. 1997;72(7):688-90.

13. Daly RC, Trastek VF, Pairolero PC, Murtaugh PA, Huang MS, Allen MS, et al. Bronchoalveolar carcinoma: factors affecting survival. Ann Thorac Surg. 1991;51:368-77.

14. Dumont P, Gasser B, Rouge C, Massard G, Wihlm JM. Bronchoalveolar carcinoma: histopathologic study of evolution in a series of 105 surgically treated patients. Chest. 1998;113:391-5.

15. Greco RJ, Steiner RM, Goldman S, Cotler H, Patchefsky A, Cohn HE. Bronchoalveolar cell carcinoma of the lung. Ann Thorac Surg. 1986; 41:652-6.

16. Okubo K, Mark EJ, Flieder D, Wain JC, Wright CD, Moncure AC, et al. Bronchoalveolar carcinoma: clinical, radiologic, and pathologic factors and survival. J Thorac Cardiovasc Surg. 1999;118(4):702-9.

17. Callejas M, Grau JJ, Belda X, Gimferrer JM, Canalis E, Catalan M, et al. Outcome of patients after curative resection of pulmonary metastases. Int Surg. 1998;83:8-10.

18. Girard P, Pierre B, Chevalier TL, Lemoine G, Tremblay C, Spielmann $\mathrm{M}$, et al. Surgical resection of pulmonary metastases up to what number? Am J Respir Crit Care Med. 1994;149:469-76.

19. Khan JH, McElhinney DB, Rahman SB, George TI, Clark OH, Merrick SH. Pulmonary metastases of endocrine origin: the role of surgery. Chest. 1998;114(2):326-34.

20. Moores DWO. Pulmonary metastases revisited. Ann Thorac Surg. 1991;52:178-9.

21. Yano T, Fukuyama Y, Yokoyama H, Tanaka Y, Miyagi J, Kuninaka S, et al. Failure in resection of multiple pulmonary metastases from colorectal cancer. J Am Coll Surg. 1997;185:120-2.

22. Albertine KH, Wiener-Kronish JP, Roos PJ, Staub NC. Structure, blood supply, and lymphatic vessels of the sheep's visceral pleura. Am J Anat. 1982;165:277-94.

23. Peyman GA. Vitreous substitutes. East Norwalk, Conn: Appleton and Lange; 1995. 